\title{
Editorial: Context-Aware Systems and Applications (ICCASA 2015)
}

\author{
Phan Cong Vinh ${ }^{1} \cdot$ Leonard Barolli $^{2} \cdot$ Emil Vassev $^{3}$
}

Published online: 21 January 2017

(C) Springer Science+Business Media New York 2017

\section{Foreword}

This special issue, with six papers invited and extended from selected contributions to The 4th International Conference on Context-Aware Systems and Applications (ICCASA 2015) held in Vung Tau City of Vietnam, will serve as a reference material for researchers, scientists, professionals and students in computer science and computer engineering as well as developers and practitioners in computing and networking systems design by providing them with state-of-the-art research findings and future opportunities and trends. These papers include some various aspects of context-awareness reflected in this special issue. In particular, the special issue covers various contributions of context-awareness as follows:

Paper 1 by Ijaz Uddin, Abdur Rakib and Hafiz Mahfooz Ul Haque presents a systematic framework to modelling and implementation of resource-bounded multi-agent contextaware systems on Android devices. The proposed framework makes use of semantic technologies for context modelling and reasoning about resource-bounded context-aware agents, Android powered smartphones as development platform, a

Phan Cong Vinh

pcvinh@ntt.edu.vn

Leonard Barolli

barolli@fit.ac.jp

Emil Vassev

emil.vassev@lero.ie

1 Nguyen Tat Thanh University, 300A Nguyen Tat Thanh Street, Ward 13, District 4, Ho Chi Minh City, Vietnam

2 Fukuoka Institute of Technology, 3-30-1 Wajiro-Higashi, Higashi-Ku, Fukuoka 811-0295, Japan

3 University of Limerick, Tierney Building, Limerick, Ireland suitable communication model and declarative rule-based programming as a preferred development language.

Paper 2 by Alistair Morris, Constantinos Patsakis, Melanie Bouroche and Vinny Cahill describes Adaptive Context Tries (ACT), a decentralised context dissemination middleware that balances message transmission and control overhead to support urban-scale context-aware applications. ACT achieves scalability using a dynamically constructed virtual overlay, structured as a retrieval tree (trie) on node identifiers (IDs), avoiding continuous overlay rebuilds due to mobility or nodes changes by removing the need for subscriptions. Through formal analysis and extensive large-scale simulations authors show that unlike existing context dissemination algorithms ACT can handle dynamic context requirements in urban-scale scenarios.

Paper 3 by Wanling Xie, Huibiao Zhu, Xi Wu, Shuangqing Xiang, Jian Guo and Phan Cong Vinh uses Communicating Sequential Processes (CSP) to model and analyze Hadoop Distributed File System (HDFS). Authors mainly focus on the dominant parts which include reading files and writing files in HDFS and formalize them in detail. Moreover, authors also model the heartbeat mechanism. Finally, authors use the model checker Process Analysis Toolkit (PAT) to simulate the model constructed and verify whether it caters for the specification and some important properties, which include Deadlock-freeness, Minimal Distance Scheme, Mutual Exclusion, Write-Once Scheme and Robustness.

Paper 4 by Francesco Piccialli and Angelo Chianese presents a Internet of Things (IoT) context-aware system and its application in the Cultural Heritage domain. Cultural Heritage is a domain where the widespread diffusion of ICT technology and sensors, within its spaces like museums, art exhibition, etc., can strongly enhance the quality of the offered services and subsequently create a Smart Cultural Environment. 
Paper 5 by A. Drif, A. Boukerram, Y. Slimani and S. Giordano considers to discover communities of mobile users and studying how do communities provide accurate knowledge to analysis the different forms of human mobility. Basic idea of the work is to model the behaviour of users with strong social characteristics regarding the context of their location histories to explore a similar interest of people by mining their mobiles communities. The proposed analysis illustrates in what way a common interest of a group of individuals can create better understanding of human mobility. Realistic models based on these interest based communities can be the basis for applications as recommendation system or wireless networks management.

Paper 6 by Hong Anh Le, Thi-Huong Dao and Ninh-Thuan Truong proposes a new approach to checking consistency between original software system and its evolution at both design and implementation phases. First, authors formalize elements of software designs and programs. Methods, based on these formalizations, are proposed for verifying the design and implementation of the system. Finally, the paper presents a case study of Adaptive Road Traffic Control system to illustrate the proposed approach in detail.

We owe our deepest gratitude to Prof. Nguyen Manh Hung - Chairman and Rector of Nguyen Tat Thanh University in Vietnam for his useful support, especially to all the authors for their valuable contribution to this special issue and their great efforts, and also to the referees for ensuring the high quality of the material presented here. All of them are extremely professional and cooperative. We wish to express our thanks to the Editor-in-Chief, Prof. Imrich Chlamtac, for his important assistance with the process of assembling the special issue.

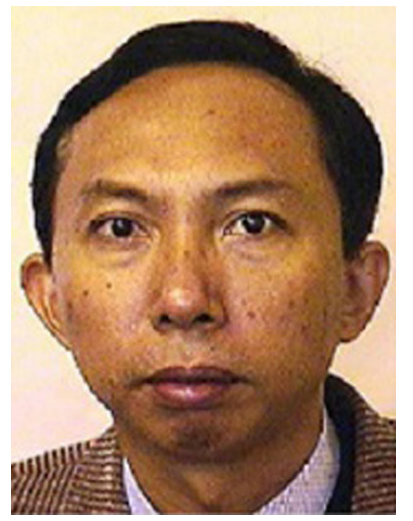

Phan Cong Vinh received a $\mathrm{PhD}$ in computer science from London South Bank University (LSBU) in the United Kingdom. He finished his $\mathrm{PhD}$ dissertation with the title of "Formal Aspects of Dynamic Reconfigurability in Reconfigurable Computing Systems" at LSBU where he was affiliated with the Center for Applied Formal Methods (CAFM) at the Institute for Computing Research (ICR). At present, he is an Associate Professor of Nguyen Tat Thanh University (NTTU) to take on the responsibility of a senior research scientist. He has been author or co-author of many refereed contributions published in prestigious journals, conference proceedings or edited books. He is editor of two books titled, "Autonomic Networking-on-Chip: Bio-Inspired Specification, Development and Verification" (CRC Press, 2012) and "Formal and Practical Aspects of Autonomic Computing and Networking: Specification, Development and Verification" (IGI Global,
2011). He has served on many conference program committees and has been general or technical (co)chair and (co)organizer of several international conferences such as ICCASA and ICTCC series. His research interests center on all aspects of formal methods, nature of computation and communication, and applied categorical structures in computer science.

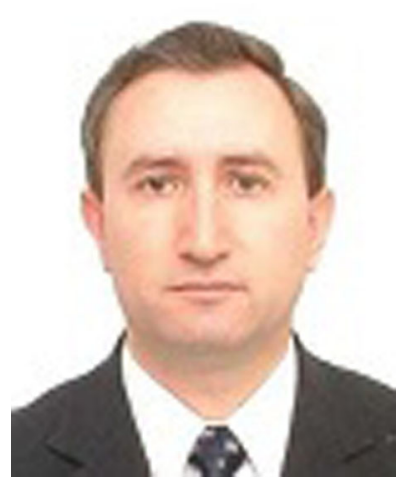

Leonard Barolli received BE and $\mathrm{PhD}$ degrees from Tirana University and Yamagata University in 1989 and 1997, respectively. From April 1997 to March 1999, he was a JSPS Post Doctor Fellow Researcher at Department of Electrical and Information Engineering, Yamagata University. From April 1999 to March 2002, he was as a Research Associate at the Department of Public Policy and Social Studies, Yamagata University. From April 2002 to March 2003, he was an Assistant Professor at Department of Computer Science, Saitama Institute of Technology (SIT). From April 2003 to March 2005, he was an Associate Professor and presently is a Full Professor, at Department of Information and Communication Engineering, Fukuoka Institute of Technology (FIT). Prof. Barolli has published more 700 papers in referred Journals, Books and International Conference proceedings. He was an Editor of the IPSJ Journal and has served as a Guest Editor for many International Journals. Dr. Barolli has been a PC Chair and General Chair of many International Conferences. Prof. Barolli is the Steering Committee Chair of CISIS and BWCCA International Conferences and Steering Committee Co-Chair of AINA, NBiS, 3PGCIC, EIDWT, INCoS and IMIS. He is organizers of many International Workshops. Prof. Barolli has won many Awards for his scientific work and has received many research funds. He got the "Doctor Honoris Causa" Award from Polytechnic University of Tirana in 2009. His research interests include network traffic control, fuzzy control, genetic algorithms, agent-based systems, ad-hoc networks, sensor networks, sensor-actor networks, P2P systems, vehicular networks, cellular networks, Web applications and medical applications. He is a member of SOFT, IPSJ, IEEE and IEEE CS.

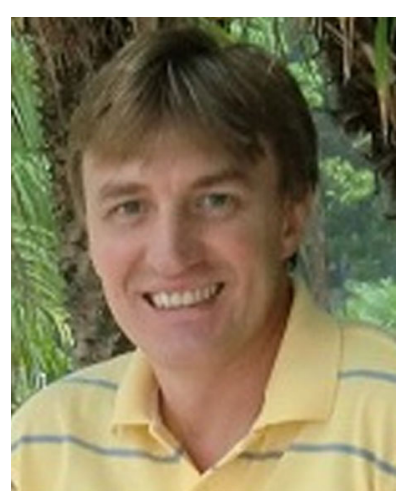

Dr. Emil Vassev received his M.Sc. in Computer Science (2005) and his Ph.D. in Computer Science (2008) from Concordia University, Montreal, Canada. Currently, he is a research fellow at Lero (the Irish SoftwareEngineering Research Centre) at University of Limerick, Ireland where he is leading the Lero's participation in the ASCENS FP7 project and the Lero's joint project with ESA on Autonomous Software Systems Development Approaches. His research focuses on knowledge representation and awareness for self-adaptive systems. A part from the main research, Dr. Vassev's research interests include engineering autonomic systems, distributed computing, formal methods, cyber-physical systems and software engineering. He has published two books and over 100 internationally peerreviewed papers. As part of his collaboration with NASA, Vassev has been awarded one patent with another one pending. 